\title{
KvarQ: targeted and direct variant calling from fastq reads of bacterial genomes
}

Andreas Steiner ${ }^{1,2+}$, David Stucki ${ }^{1,2+}$, Mireia Coscolla ${ }^{1,2}$, Sonia Borrell ${ }^{1,2}$ and Sebastien Gagneux ${ }^{1,2^{*}}$

\begin{abstract}
Background: High-throughput DNA sequencing produces vast amounts of data, with millions of short reads that usually have to be mapped to a reference genome or newly assembled. Both reference-based mapping and de novo assembly are computationally intensive, generating large intermediary data files, and thus require bioinformatics skills that are often lacking in the laboratories producing the data. Moreover, many research and practical applications in microbiology require only a small fraction of the whole genome data.

Results: We developed KvarQ, a new tool that directly scans fasta files of bacterial genome sequences for known variants, such as single nucleotide polymorphisms (SNP), bypassing the need of mapping all sequencing reads to a reference genome and de novo assembly. Instead, KvarQ loads "testsuites" that define specific SNPs or short regions of interest in a reference genome, and directly synthesizes the relevant results based on the occurrence of these markers in the fastq files. KvarQ has a versatile command line interface and a graphical user interface. KvarQ currently ships with two "testsuites" for Mycobacterium tuberculosis, but new "testsuites" for other organisms can easily be created and distributed. In this article, we demonstrate how KvarQ can be used to successfully detect all main drug resistance mutations and phylogenetic markers in 880 bacterial whole genome sequences. The average scanning time per genome sequence was two minutes. The variant calls of a subset of these genomes were validated with a standard bioinformatics pipeline and revealed $>99 \%$ congruency.

Conclusion: KvarQ is a user-friendly tool that directly extracts relevant information from fastq files. This enables researchers and laboratory technicians with limited bioinformatics expertise to scan and analyze raw sequencing data in a matter of minutes. KvarQ is open-source, and pre-compiled packages with a graphical user interface are available at http://www.swisstph.ch/kvarq.
\end{abstract}

Keywords: Whole genome sequencing, FastQ, Single nucleotide polymorphisms, In-silico SNP-typing, Mycobacterium tuberculosis

\section{Background}

Large-scale whole-genome sequencing (WGS) is revolutionizing microbiology and public health [1-3]. Thanks to the latest technological advances, bacterial genomes can now be sequenced in less than 48 hours for less than $€ 100$ per genome. New benchtop devices are providing WGS capability to routine microbiological laboratories, and WGS is now becoming an important part of clinical microbiology and molecular epidemiology. This emerging field of "genomic epidemiology" [4-11] already comprises

\footnotetext{
* Correspondence: sebastien.gagneux@unibas.ch

${ }^{\dagger}$ Equal contributors

'Swiss Tropical and Public Health Institute, Socinstrasse 57, Basel 4051, Switzerland

${ }^{2}$ University of Basel, Basel, Switzerland
}

numerous studies that have evaluated the potential of WGS to trace the transmission of pathogens such as Clostridium difficile, Pseudomonas aeruginosa, Klebsiella pneumonia, Neisseria meningitidis, Staphylococcus aureus and Mycobacterium tuberculosis [12-21]. Moreover, WGS is playing an increasing role in molecular drug susceptibility testing (DST) and the identification of drug resistance mutations [22-28]. It is expected that WGS will eventually replace all other bacterial genotyping methods [29].

In contrast to the increasing availability of WGS platforms, the capability to analyse WGS data is lagging behind, partially because of the lack of rapid and user-friendly analysis tools. Extracting single nucleotide polymorphisms (SNPs), the most widely studied form of genetic variation, from WGS data requires substantial bioinformatics expertise, as 
well as dedicated bioinformatic analysis pipelines often based on customized scripts. Moreover, this standard approach of analysing WGS data is time-consuming and computationally expensive [30], and as a consequence, biological questions can often not be addressed directly by the people generating the data. Furthermore, large amounts of data are produced when analyzing complete genomes, although often only a small proportion of these data are relevant for the study question; this is particularly true when screening for drug resistance determinants. Identifying individual SNPs of interest usually requires mapping all sequencing reads in a fastq file to a given reference genome, despite the fact that only a few nucleotide positions might be relevant. Tools have been developed to speed up the extraction of relevant information. These include in silico multi-locus sequence typing (MLST) from draft genomes or contigs, but all these methods rely on previous assembly [31-33]. One of these tools, SRST, facilitates the process by accepting fastq files for a given typing scheme. SRST uses BWA and SAMtools and is highly configurable, but is aimed at the bioinformatically skilled users and lacks a graphical interface. However, a graphical interface is crucial for making these kinds of genome analyses accessible to a broader group of microbiologists, including the ones primarily interested in routine clinical applications. Consequently, there is a need for software able to extract allelic information directly from the raw sequencing reads without the need of previous mapping or de novo assembly.

Here we present KvarQ, a software that enables rapid screening of short sequence reads for mutations at multiple nucleotide positions of interest. KvarQ uses as input a fastq file, and generates the output in form of a text file in JavaScript Object Notation (json) format. Using a complete bacterial genome sequence as a reference, KvarQ can interrogate any single nucleotide position or short DNA sequence of interest for known or unknown mutations, respectively.

KvarQ is universally applicable to any short read dataset and corresponding reference sequence, and is highly adaptable with respect to target polymorphisms. Clinical microbiological laboratories will benefit from KvarQ's short analysis time, while laboratories in resource-limited settings can get access to genome analysis despite the lack of advanced computational infrastructure and/or appropriately skilled staff.

Even advanced users will save time by avoiding the need for formal mapping or assembly to answer specific questions, particularly when studying large numbers of genomes.

KvarQ is available for Linux, OS X and Windows, and runs on any portable or desktop system. A command line interface and a simple graphical user interface are available. We aimed at a self-explaining application with short run times $(<20$ minutes on a standard workstation for a fastq file of $1000 \mathrm{MB}$ ), while keeping the program flexible enough to be used with other organisms. KvarQ can be downloaded (source code as well as precompiled packages) from http://www.swisstph.ch/kvarq.

\section{Implementation \\ Overview}

KvarQ analyzes a fastq file and detects the allelic state of known polymorphisms, and compiles all the relevant information about the genome sequence. In contrast to current genetic analysis software [31,33], KvarQ extracts the relevant information directly from the sequencing reads, without the need to map every read to a reference genome. The software can be used via the command line or the graphical user interface, and is split into one part that scans the fastq files and saves the results to a json file, and a second part that extracts and illustrates the information contained in the json file. Although results reported in this article mainly use sequencing data from the Mycobacterium tuberculosis complex (MTBC), the software can be used with any short read sequencing data from any organism. Currently, KvarQ is optimized for haploid organisms, but could be extended to analyze diploid data sets. In principle, KvarQ could be used to scan through very large datasets, such as from human genomes, although the scan time would increase linearly with data size.

\section{Algorithm and parameters}

The following paragraphs describe the information flow inside KvarQ from the fastq files to the final results that are displayed to the user (Figure 1).

Before the scanning process, target sequences are generated from known mutations or regions of interest. KvarQ uses a modular approach, where different "testsuites" (realized as python modules) define known single nucleotide polymorphisms (SNPs) or small regions of interest that harbour potential mutations (such as the Rifampicin Resistance-Determining Region, RRDR, in MTBC [34]). The target genomic sequence is extracted from a reference genome and flanked on both sides with additional bases. These flanking regions increase the length of the sequence and thereby increase the coverage over the region of interest (see Figure 2). For each of these "target sequences", the corresponding base-sequence on the complementary strand is also generated.

The actual scanning algorithm is implemented in $\mathrm{C}$ and splits the fastq file into small parts that are distributed to multiple threads and scanned simultaneously. First, every read is trimmed by a user-specified minimum quality score. The trimmed part is then shifted along each of the target sequences and every match (not exceeding a specified number of single nucleotide differences) is recorded in the "hit-list" if a minimum overlap is warranted. Currently, no 


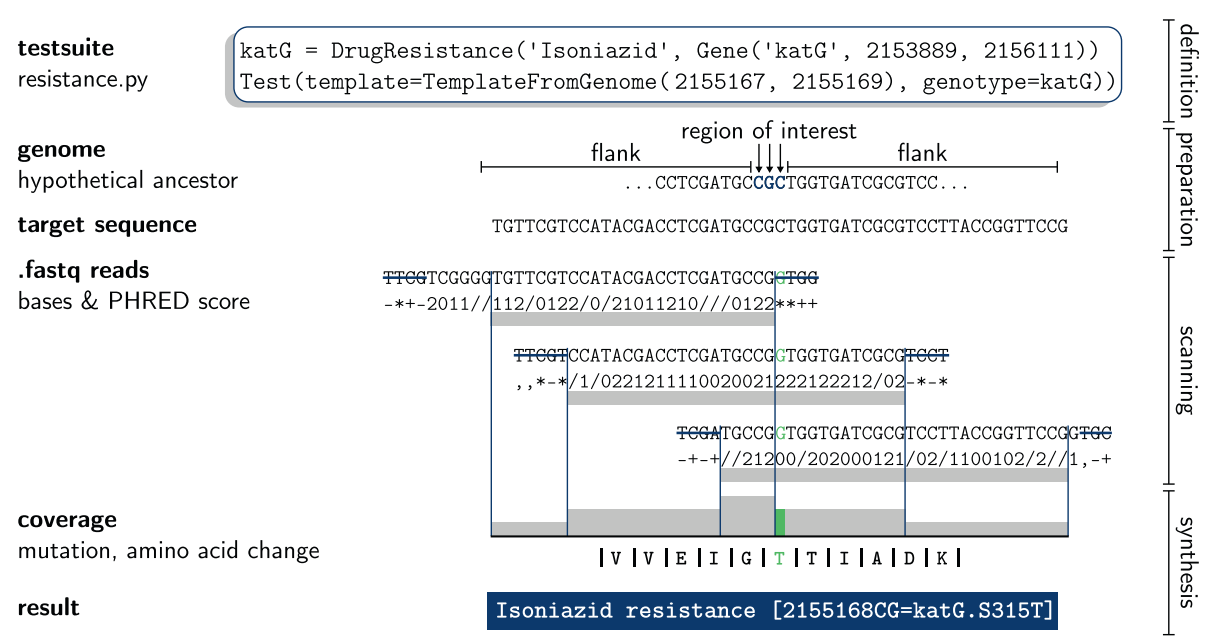

Figure 1 Simplified overview of scanning process and preparation. "Testsuites" are python source files that define the SNPS of interest (or in this case a 3 base pair long region) as well as other relevant genetic information (in this case the katG gene in which mutations can confer isoniazid resistance). This information is used to extract a "target sequence" from a reference MTBC genome: on both sides, additional bases ("flanks") are concatenated to avoid border effects within the sequence of interest. During the scanning process, every read is trimmed depending on its PHRED score (in this case, a quality cutoff of $\mathrm{Q}=13$ was defined which corresponds to the ASCII character " $/$ "). After the scanning, the part of the reads that matched the target sequence and exceeded the minimum quality score (represented with gray bars) are assembled to a "coverage" that indicates the overall coverage depth as well as all detected mutations (green). In a further step, additional information is generated from this coverage (such as the resulting amino acid sequence) and finally a short "result" string is generated that summarizes the result of the scanning process.

indexing/caching is used for comparison of the reads with the target sequences. This works fast enough for the short target sequences used in our analysis. The scanning process continues until the whole file is scanned or a specified minimum coverage is reached.

Next, the data gathered from the fastq files is translated into intermediary data structures ("coverages"). These data structures combine the reads from the original as well as the complementary strand, position them within the genome, and summarize non-matching bases in reads (these can be true mutations in the genome or sequencing errors).

Finally, the testsuites determine the results based on the information conveyed by the intermediary data structures created in the last step. Currently, mutations are extracted by using a simple threshold that separates them from sequencing errors - this is possible because KvarQ neglects bases that do not satisfy the minimum read quality

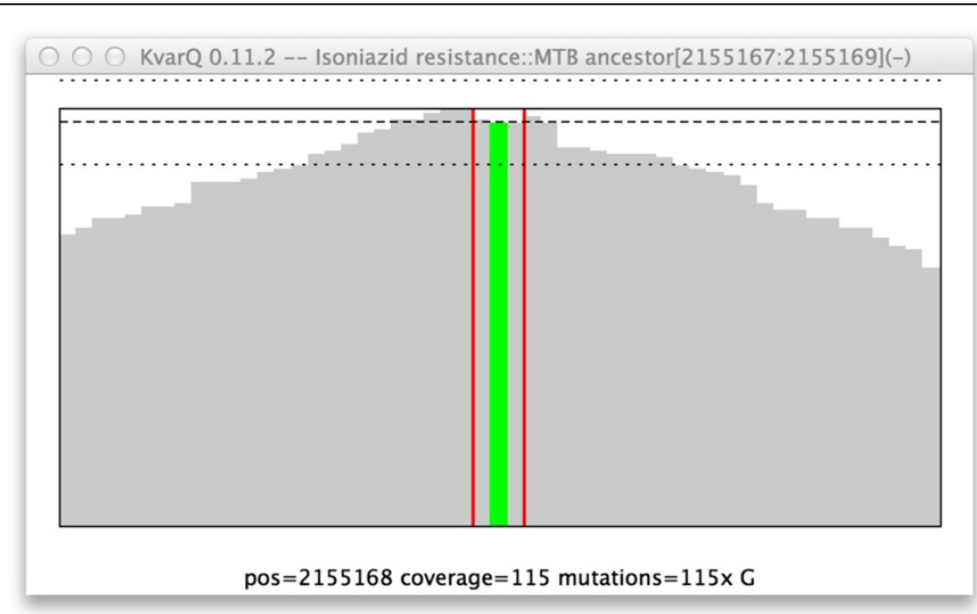

Figure 2 Interactive inspection of "coverage". This screenshot shows the coverage over a short region of interest (3 base pairs within vertical red lines, corresponding to codon 315 in $\mathrm{katG}$ ) and the surrounding flanking sequence. Note the mutation in the middle of the three base pair long sequence (in green, text description at bottom of window). The decrease of coverage on both sides is due to the minimum overlap that is set to a value equal to the length of the two flanking regions. 
requirements set by the user. How this mutation information is processed further varies depending on the various testsuites. For example, the phylogenetic testsuite we applied for MTBC uses three phylogenetically informative SNPs for every lineage and imposes logical constraints on sublineages. For example, any genome belonging to the MTBC "Beijing" sublineage must also have the SNPs characteristic of "Lineage 2". The resistance testsuite checks for every mutation whether a change in amino acid sequence follows the nucleotide change (non-synonymous mutations) and only reports those to the user. If the results have a low confidence due to low coverage of the sequences, this is also added as a remark to the result output.

\section{Data format and analysis}

KvarQ uses fastq files with Solexa, Sanger and different Illumina quality scores as input files. The quality format is determined by a heuristic search. Only one input file per genome sequence is accepted, and paired-end files are automatically included. The output is structured data in json format, a human readable file that contains information about the scanning process (parameters used, fastq file size, statistics about read number, length and quality, and scan time), the final results, and the intermediary data structures that were used to calculate these results. The information contained in the json file can be used to re-calculate the results without the need to re-scan the fastq file, because most of the relevant information is contained in the intermediary data structures that are saved along with the results in the json file.

To facilitate the extraction of relevant results, KvarQ provides data analysis tools to inspect the data interactively from the command line or with a hierarchical menudriven graphical user interface. The "json explorer" shows the summarized test results for every testsuite (KvarQ's main goal is to be user-friendly) as well as detailed information about the coverage of every target sequence that was used by the different testsuites (Figures 2 and 3). The interactive exploration of this wealth of information is intended for the advanced operator to get, for example, a better impression of the usefulness of newly designed target sequences or the fastq quality. Additionally, the "json explorer" displays overall number of reads in a fastq file and the length of the reads.

\section{Results}

We validated KvarQ with 880 fastq files of predominantly Mycobacterium tuberculosis complex (MTBC) isolates, for which we interrogated 206 genomic positions where polymorphisms have been previously described (Additional files 1 and 2).

In MTBC, single nucleotide mutations are reliable drug resistance markers, as most phenotypic drug resistance is conferred by single amino acid changes [34-36]. Hence, molecular DST is becoming increasingly widespread in the diagnosis of $\mathrm{TB}$, and various genotyping platforms make use of these markers. Moreover, SNPs are powerful phylogenetic markers in MTBC, because similar to other genetically monomorphic bacteria, MTBC exhibits limited horizontal gene exchange and as a consequence, SNP homoplasies are rare $[37,38]$. Hence, SNPs can be used to study the evolutionary history of MTBC, as well as to classify clinical strains associated with variable degrees of virulence or different clinical outcomes [39,40]. MTBC comprises several phylogenetic lineages, and SNPs represent ideal markers to classify clinical MTBC isolates into these lineages $[41,42]$.

In this study, we included 27 SNPs as phylogenetic markers defining MTBC lineages and sublineages, as well as 32 single nucleotide mutations and three genomic regions (63 base pairs (bp) in $g y r A, 81$ bp in $r p o B$ and 3 bp in $k a t G$ ) associated with drug resistance (Additional file 1).
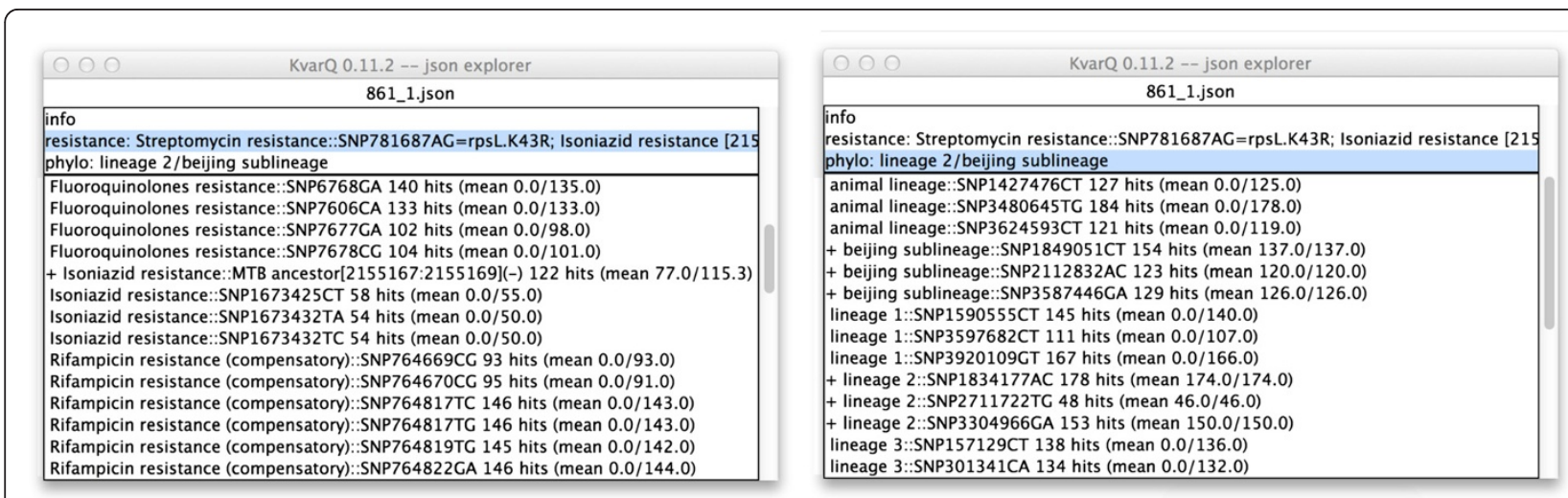

Figure 3 Interactive inspection of json file. The upper pane of each window in these screenshots shows the main categories of data contained in the json file. In the left window, the drug resistance section is selected and the lower pane shows details about all target sequences in this testsuite (the " $+"$ in front of the "Isoniazide resistance" indicates a non-synonymous mutation). In the right window, the phylogenetic section is selected, showing that all SNPs for "lineage 2" and "beijing sublineage" were found. 
We tested KvarQ with a set of "in-house" generated whole genome sequences of clinical MTBC isolates and additionally downloaded sequences from public sequence read archives (Additional file 2). Different, overlapping subsets of fastq files were used for $i$ ) the comparison of SNP-calls with a standard BWA-based mapping pipeline $(\mathrm{N}=206)$, ii) detecting drug resistance mutations $(\mathrm{N}=19)$ and iii) comparison of KvarQ phylogenetic classification with laboratory based classification $(\mathrm{N}=321)$ - see Figure 4. In addition to these wellcharacterized genome subsets, we used a previously published "blind" subset of 388 fastq files downloaded from a public source to extract phylogenetic information and drug resistance mutations, which were not reported in the original publication [21].

\section{KvarQ scanning times and overall performance}

First, we applied KvarQ to all 880 fastq files and calculated scanning times for the set of SNPs described. The scanning times were found correlated with the average genome coverage (or the total base output) in the fastq file. A genome sequence file with 100-fold coverage of the MTBC genome required approximately 2 minutes of KvarQ scanning time, and increased linearly with sequence coverage (Figure 5).
To assess overall performance, we looked at phylogenetic classifications in all 880 fastq files. These files included whole genome sequences generated on different sequencing devices using different laboratory procedures (i.e. library preparations), and of unknown quality (Additional file 2). Successful phylogenetic classification was obtained for 865/880 fastq files (98.3\%) (Figure 6). The remaining 15 included the chimpanzee bacillus [43], Mycobacterium canettii and eight confirmed non-MTBC isolates, for all of which no MTBC lineage classification would be expected. For five other strains, no MTBC lineage-specific SNP was detected, which can be due to low coverage or because isolates were not MTBC.

Furthermore, we compared the number of KvarQ hits per position with overall base output in the fastq file (data not shown). The correlation varied strongly between positions on the genomes and between fastq files, which indicates that the number of hits per SNP depends on the SNP queried (i.e. the particular genomic region being interrogated) and the quality of the base calls in the fastq file.

\section{Comparison of SNP-calls with BWA calls}

A subset of 206 FastQ files was used to directly compare SNP-calls obtained with KvarQ with SNP-calls generated with our standard pipeline using BWA, SAMtools, BCFtools

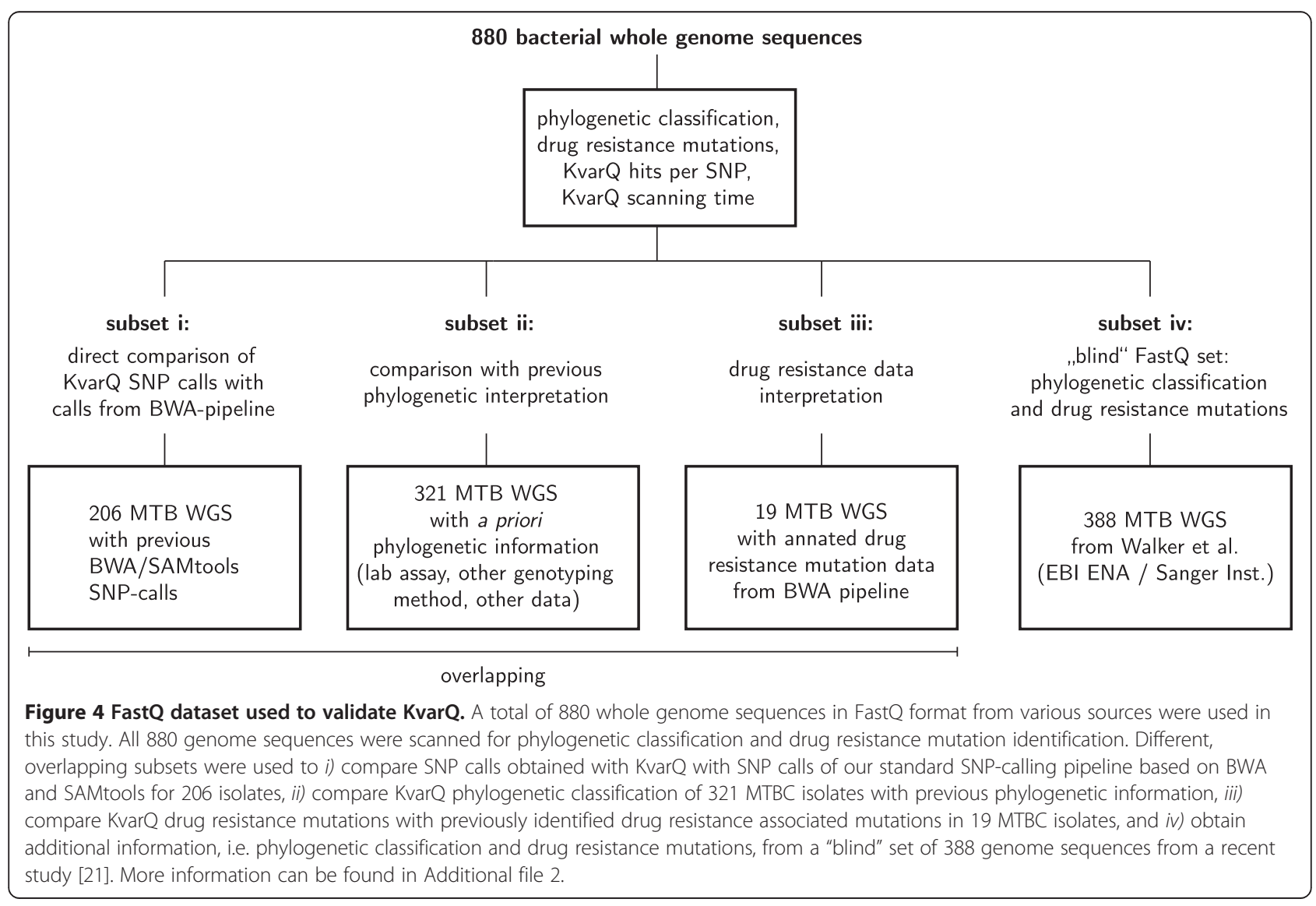




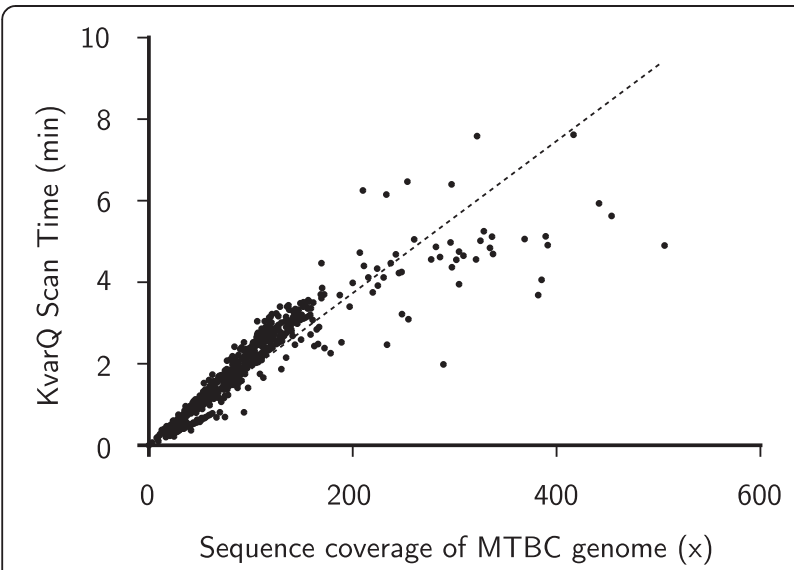

Figure $\mathbf{5}$ KvarQ scanning time for all 206 polymorphic positions with respect to average sequence coverage of the MTBC genome per fastq file. Each dot represents one isolate (i.e. fastq file). Scanning time was found to be 112 seconds/100x coverage. Eleven isolates of 880 were excluded due to variable read lengths (difficult calculation of total base output). Three additional isolates were excluded because of file sizes larger than $10 \mathrm{~GB}$.

and custom scripts for filtering [43]. For each of the 206 positions (single nucleotide positions plus regions of interest), the BWA list of variants in the corresponding genome was interrogated for presence of an alternative allele compared to the reference genome (reconstructed hypothetical ancestor $[38,41])$. With this, a direct comparison of all 206 positions in 206 strains was performed, resulting in 42,436 data points (Table 1).

A total of 782 mutations were called by both KvarQ and the BWA-pipeline ( $1.8 \%$ of all data points). This corresponds to a sensitivity of $99.2 \%$ in mutation calling of KvarQ (782/788) taking the BWA pipeline as the gold standard. Specificity was found to be $99.9 \%(41,615 / 41,648$ positions).

Thirty-three mutations $(0.08 \%$ of all data points) in 20 fastq files were called by KvarQ, but no mutation was found in the corresponding BWA SNP-list. Upon manual inspection, we found a successful SNP-call in the SAMtools pileup-list for each of the 33 mutations, but these mutations were filtered out in the subsequent heuristic filtering step including the SAMtools varFilter command for unknown reasons and despite good quality and coverage.

We found three fastq files where KvarQ missed a single SNP and one file where KvarQ missed 3 SNPs despite six calls in the BWA-pipeline. The BWA read depths for these six calls $(0.01 \%$ of all data points) were $5,6,21,38$, 64 and 77. Corresponding KvarQ number of hits were 0 , 1, 2, 3, 4 and 5, respectively. For strain MTB_erdman_SRR017677, when the KvarQ quality cutoff was removed (all reads considered), the number of hits increased to 57, 84 and 120, respectively. Subsequent FastQC analysis (http://www.bioinformatics.babraham.ac. uk/projects/fastqc/) showed poor fastq quality over the whole read length (data not shown).

\section{Validation of phylogenetic classification}

A subset of 321 isolates was used to compare the phylogenetic classification by KvarQ with data obtained previously by various genotyping methods (molecular assays in our laboratory [40]), data obtained from the collaborating laboratory that provided DNA for WGS, or metadata from the sequence read archive in the case of downloaded files. The comparison included seven main phylogenetic lineages [41], the animal-associated MTBC clade (M. bovis/M. caprae), the Beijing sublineage of Lineage 2, and 10 nonMTBC isolates. In 309/321 (96.3\%) isolates, KvarQ detected MTBC lineage-specific SNPs that were in agreement with the previous classifications (Additional file 2). The remaining 12 genome sequences for which no MTBC lineage-specific SNP was found included eight nonMTBC, the chimpanzee bacillus and M. canettii, for

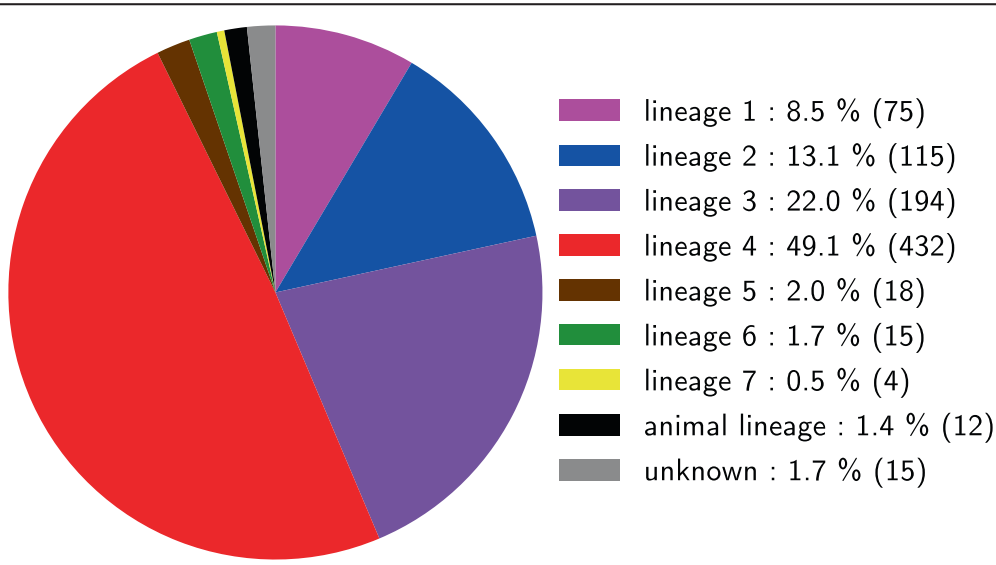

Figure $\mathbf{6}$ Phylogenetic classification of all $\mathbf{8 8 0}$ isolates used in this study. This figure shows the distribution of phylogenetic markers in all of the scanned genomes. For 865 isolates, an MTBC lineage-specific SNP was found. In 15 isolates, no MTBC lineage-specific SNPs were found, either because isolates were non-MTBC (10 were known to be non-MTBC), or because coverage was low. 
Table 1 Comparison of SNP-calls at 206 polymorphic positions in 206 MTBC genome sequence fastq files

\begin{tabular}{|c|c|c|c|c|c|c|}
\hline & \multicolumn{4}{|c|}{ SNP in BWA mapping pipeline } & & \\
\hline & \multicolumn{2}{|c|}{ Yes } & \multicolumn{2}{|c|}{ No } & \multicolumn{2}{|c|}{ Total } \\
\hline & $\mathbf{N}$ & $\%$ & $\mathbf{N}$ & $\%$ & $\mathbf{N}$ & $\%$ \\
\hline SNP in KvarQ & 782 & 99.24 & 33 & 0.08 & 815 & 1.92 \\
\hline no SNP in KvarQ & 6 & 0.76 & 41615 & 99.92 & 41621 & 98.08 \\
\hline Total & 788 & 100 & 41648 & 100 & 42436 & 100 \\
\hline
\end{tabular}

which no MTBC-lineage specific SNP was expected, and two isolates $(0.6 \%)$ with low KvarQ coverage and therefore no lineage-call.

\section{Drug resistance associated mutations}

KvarQ was applied to a subset of 19 strains harbouring known mutations associated with drug resistance to validate the interpretation (annotation) of the mutations. The 19 strains overlapped with the 206 strains of the direct comparison described above, but the resistance-associated mutations were identified with separate scripts from the annotated SNP-list in the BWA pipeline. The 179 drug resistance associated genomic positions were interrogated with KvarQ, including the $81 \mathrm{bp}$ Rifampicin Resistance Determining Region (RRDR) of rpoB, the $63 \mathrm{bp}$ Quinolone Resistance Determining Region of gyrA (QRDR) and $3 \mathrm{bp}$ in the codon 315 of $k a t G$ (Additional file 1).

Among the 19 strains, 16 (84\%) had perfectly congruent drug resistance mutations identified by KvarQ when compared to the BWA pipeline. For one strain (GQ1580), one mutation in rpoC was not found by KvarQ. However, this mutation was not in the list of target SNPs (Additional file 1). For one isolate, MTB_KZN_605, an additional mutation in $g y r A$ was found with KvarQ, and was not found with the BWA pipeline due to low read depth and the presence of an alternative allele. For another isolate, MTB_russia_ERR015616_1, six additional mutations in five genes were found by KvarQ. All mutations in these two isolates were traced back to the filtering problem discussed above, and were found in the pileup file.

In total, KvarQ detected 314 drug resistance associated mutations in 139/880 files (15.8\%) (Figure 7).

\section{"Blind" set of fastq files from a public repository}

Finally, a "blind" set of whole genome sequences was used to illustrate the added value provided by KvarQ. We downloaded available sequences of a recent publication that used whole genome sequencing for molecular epidemiology of MTBC transmission [21]. This publication did not report drug resistance data or phylogenetic data. We therefore analyzed all available sequences for phylogenetic SNPs and drug resistance mutations. The original sample set consisted of 390 isolates, but two fastq files were not found for download (http://www.sanger.ac.uk,
ERR192249 and ERR192250). Results for 388 isolates were obtained in less than 12 hours. A total of 62 drug resistance mutations were identified in 33 patient isolates from 11 patients (Figure 8). All paired isolates (cross-sectional: paired pulmonary and extrapulmonary isolates from the same patients; longitudinal: paired isolates from the same patient separated by at least 6 months; shown as smaller dotted boxes in Figure 8) were found to harbour identical drug resistance associated mutations, except for one patient (P000155), where an additional mutation was found in two of three isolates. Isolates of one community transmission cluster (defined by MIRU-VNTR), cluster 9 , were found to harbour drug resistance associated mutations (large box in Figure 8). Within cluster 9, all patient isolates harboured the same drug resistance mutations, except for one patient (P000179) with three otherwise unidentified mutations. Among the 388 isolates, we found a predominance of Lineage 4 (i.e. the Euro-American lineage) in the sample set $(65.5 \%)$, followed by Lineage $3(24.0 \%)$ and Lineage 1 (5.9\%) (Figure 9).

\section{Discussion}

In this article, we propose a new approach to in silico SNP-typing from whole-genome sequencing data. KvarQ scans fastq short sequence reads directly for known polymorphisms instead of mapping or assembling the complete genome. This results in a massively reduced computation time required compared to established WGS analysis tools. At the same time, KvarQ offers a high degree of flexibility and is also user-friendly. This was achieved by developing an extended command-line interface together with pre-compiled packages with a graphical user interface.

KvarQ was designed for bacterial genomes, but is applicable to any fastq sequence data. The default parameters work best for the different Illumina machines, but can easily be adapted to data from other manufacturers. For example, the Roche 454 reads in our testset were scanned with a lower quality cutoff. Due to the lack of relevant data from MTBC isolates, we were not able to test reads from Pacific Bioscience devices.

Both the reference sequence and the interrogated polymorphisms can be changed or extended. Changing the SNPs of interest can be achieved by modifying the templates in the testsuites, which are lists of nucleotide positions screened by KvarQ. An extended documentation including instructions for changing the target polymorphisms is distributed with the software and available online (http://www.swisstph.ch/kvarq). Scanning parameters are adjusted by invoking a number of options in the command-line interface or via the settings dialog in the graphical user interface.

Using our test set of 880 fastq files, we obtained KvarQ running times of less than 2 minutes on a high- 


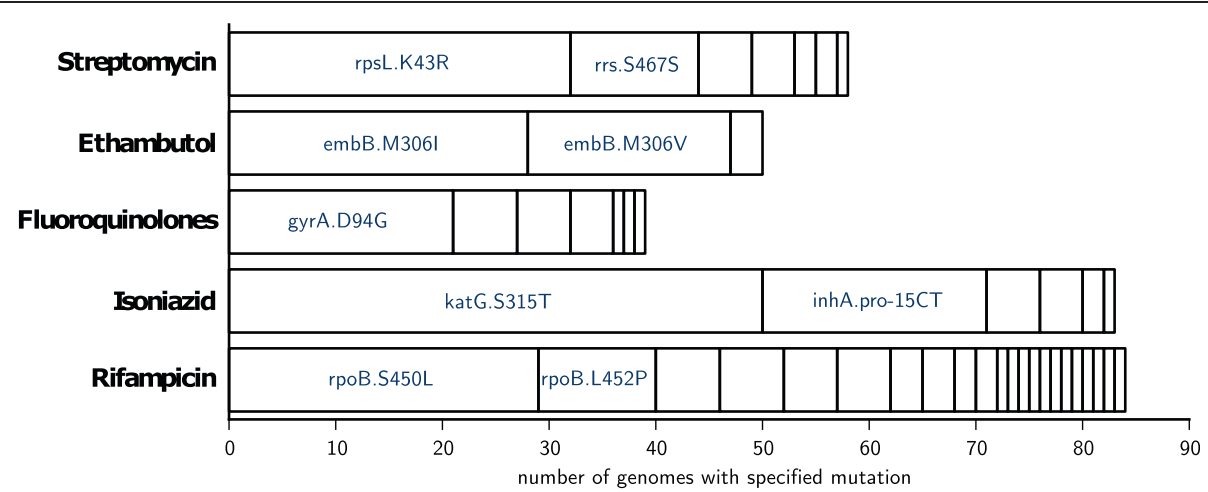

Figure 7 MTBC drug resistance associated mutations found in all $\mathbf{8 8 0}$ isolates. This figure shows all 314 mutations that were found with KvarQ in 139 isolates with at least one mutation in any of the drug resistance associated genes that were analysed (see Additional file 1). Only mutations that were found in at least ten isolates are labeled.

performance computer (average running time $110 \mathrm{sec}-$ onds) and less than 20 minutes on a standard desktop computer (Figure 5). This important decrease in time for analysis (when compared to the conventional pipeline in which the whole genome sequence is first reconstructed) is crucial for the efficient treatment of data as WGS becomes more widely available. The overall performance of KvarQ was high, as reflected by $98.3 \%$ of genome sequences successfully assigned to a phylogenetic MTBC lineage (Figure 6). By including phylogenetically redundant SNPs, the sensitivity of classification was increased. Specifically, classifications were obtained if 2 of 3 redundant SNPs were found mutant. The $96.3 \%$ congruence of lineageassignment of KvarQ with a priori lineage classification illustrates how WGS combined with a quick tool such as KvarQ has the potential to replace previous genotyping methods.

When comparing KvarQ SNP-calls with calls from our mapping pipeline using BWA and SAMtools, we found a high sensitivity and specificity of $99.2 \%$ and $99.9 \%$, respectively. The three mutations that were called by the BWA pipeline but not by KvarQ were resolved when lowering the quality cutoff parameter. FastQC (http://www.bioinformatics. babraham.ac.uk/projects/fastqc/) confirmed the low quality of the reads in these files.

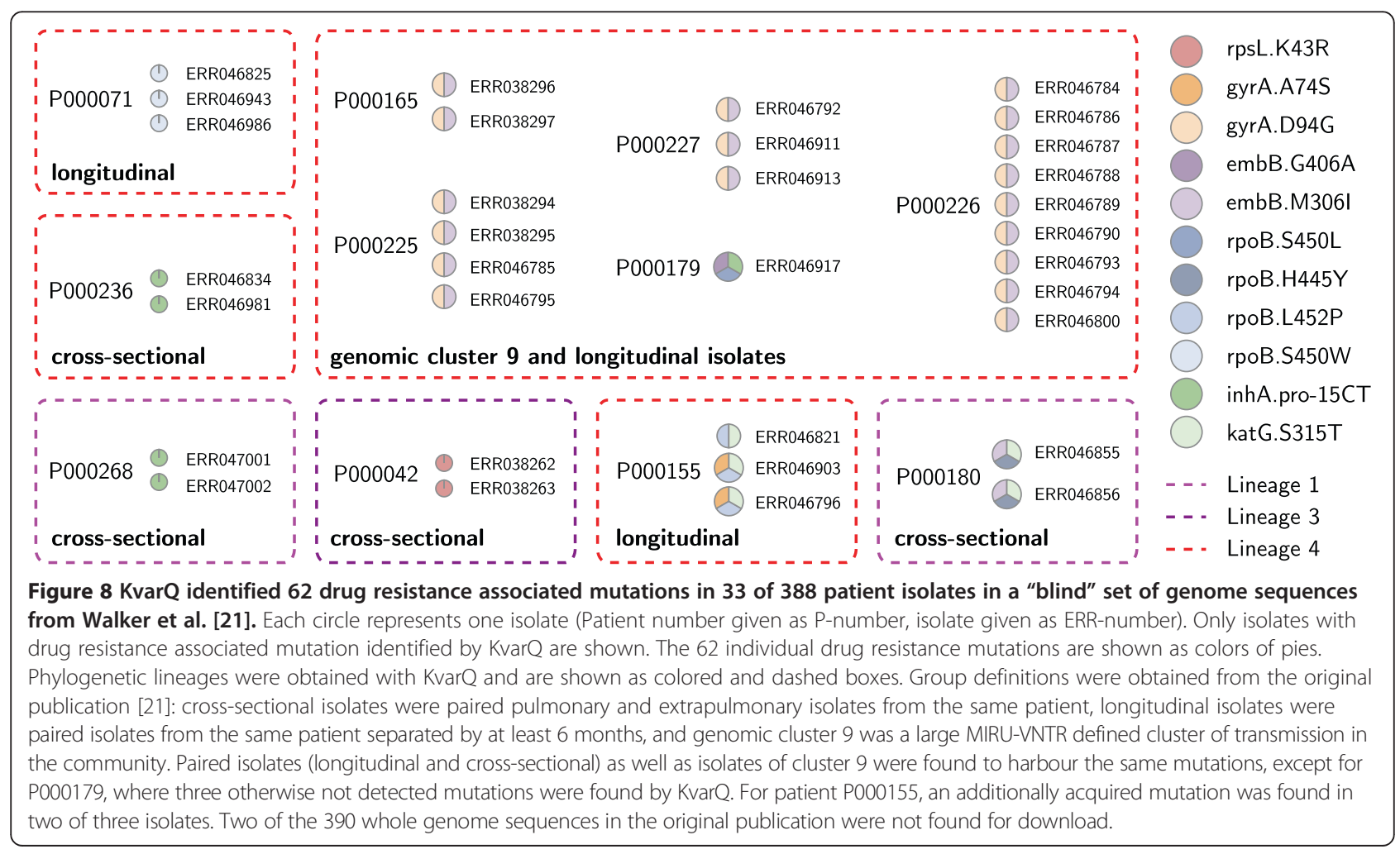




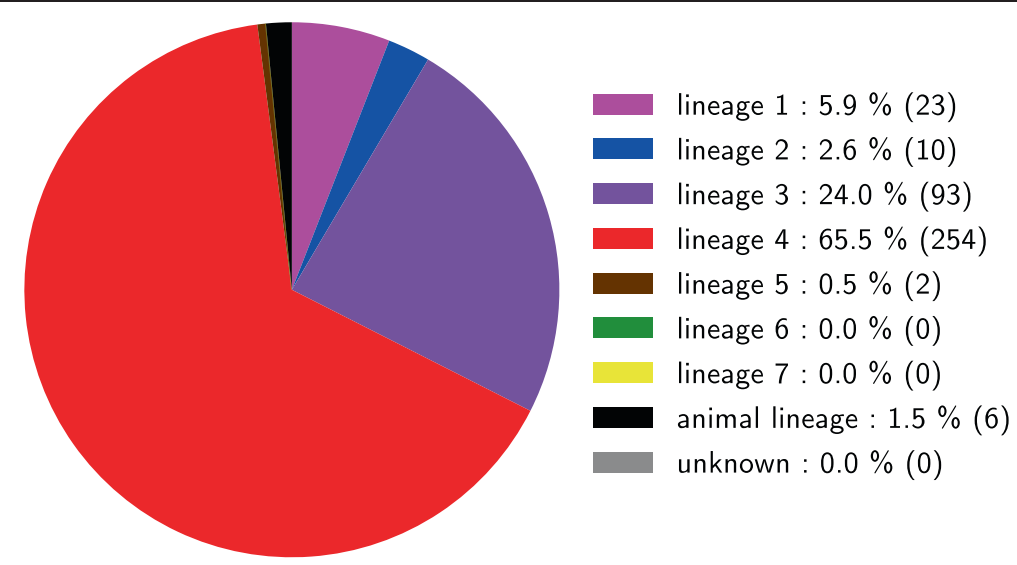

Figure 9 Main MTBC phylogenetic lineage classification of the fastq set of Walker et al. [21], consisting of 388 isolates. Successful lineage assignment was obtained for 388/388 (100\%) isolates. Figure includes all clustered isolates as well as longitudinal, cross-sectional and household isolates.

Drug resistance mutations obtained with KvarQ and BWA in the 19 compared files were nearly identical, but showed one limitation. Mutations conferring resistance to pyrazinamide are found in any part of $p n c A$, which is $561 \mathrm{bp}$ long [44]. KvarQ was designed to scan short stretches of DNA, and we therefore excluded mutations in $p n c A$ to avoid an increase in scanning time.

In the total set of 880 genomes, we found many additional drug resistance associated mutations (Figure 7) that could not be validated due to the lack of laboratory DST data for many of the isolates and the complex genetic nature of drug resistance in MTBC [45].

Successful KvarQ calls depended on the selection of suitable SNPs. Several SNPs had to be replaced in the testing phase. Most SNPs that were replaced occurred in repetitive regions, and these SNPs were also never called when using the BWA-pipeline. We therefore recommend including only SNPs that were previously found to be in core genome regions, in non-repetitive regions and where no deletions have been described.

With a "blind" set of genome sequences obtained from a recent publication [21], we were able to obtain lineage classifications and drug resistance calls, which had not been reported in the original publication, in less than one day. Using the clustering information of the publication, all isolates identified as drug-resistant by KvarQ and from the same patient (paired isolates) or the same patient cluster shared the same drug resistance mutations (except patient P000179, see Figure 8) and were assigned to the same phylogenetic lineage, highlighting the consistency in mutation calling. The fast generation of drug resistance and phylogenetic data illustrates the application of KvarQ for quick targeted scanning of large public datasets.

KvarQ was designed to interrogate positions with known mutations. However, as some regions (e.g. hot spot regions of drug resistance) can harbour several mutations in close proximity, we included the capability to scan regions of interest for the presence of any (new) mutation. These regions can be of any length, but the scanning time increases in square with sequence length. The drug resistance testsuite in the current version checks some short regions known to harbour many different drug resistance associated mutations and reports any new mutation that would result in a amino acid change of the associated gene. In future releases, KvarQ could be extended to scan for other genomic polymorphisms, such as small insertions and deletions (InDels) as well as large sequence deletions or duplications.

\section{Conclusion}

In conclusion, KvarQ provides a user-friendly and highly flexible platform for rapid and targeted analysis of fastq files. KvarQ will help overcome the hurdles of whole genome sequence analysis in clinical microbiological laboratories and other settings where bioinformatics capacity is limited. The short running times, the userfriendly graphical interface and the high configurability at the command line level allows analysing hundreds of fastq files in a short time.

\section{Methods}

\section{Informative SNPs for MTBC}

We used known phylogenetic markers and drug resistance associated mutations of MTBC to validate and benchmark KvarQ when scanning genome sequences of clinical MTBC strains. Phylogenetically informative SNPs were selected from previous publications $[40,42]$. For each of the seven main phylogenetic lineages of human-associated MTBC, plus the Mycobacterium bovis/M. caprae lineage, we included three redundant canonical SNPs as markers for the corresponding lineage. Previously published SNPs 
were complemented with SNPs obtained as described before [40] for cases where less than three SNPs per phylogenetic clade were available. The extraction of these additional SNPs was based on 172 genomes described by [41]. For Lineage 2, we additionally included known polymorphisms to discriminate the so-called "Beijing" lineage from non-Beijing Lineage 2 strains. An overview of the phylogenetically informative SNPs included in KvarQ is shown in Additional file 1. We included drug resistancemutations obtained from the Tuberculosis Drug Resistance Database (TBDReaMDB) [46], and additionally compensatory mutations from [47]. High-confidence mutations for the most important anti-tuberculosis drugs were selected (isoniazid, rifampicin, ethambutol, streptomycin, fluoroquinolones and second-line injectable drugs). Pyrazinamideresistance conferring mutations were excluded. Mutations in TBDReamDB are listed as codon changes, therefore we generated the corresponding nucleotide changes for inclusion in KvarQ. For rifampicin- and fluoroquinoloneresistance conferring mutations, we included the rifampicin-resistance determining region (RRDR) [34] and the quinolone resistance determining region (QRDR) [48], respectively, rather than specific positions. The codon 315 of kat $G$ was also treated as a region of three base pairs. All included mutations and regions associated with drug resistance mutations are listed in Additional file 1. All genomic positions given in this study refer to the genome of the strain H37Rv (NC000962.3/AL123456.3) [44,49,50].

\section{Short-read datasets}

We used a test set of 880 bacterial whole genome sequences in fastq format to screen for mutations with KvarQ. This set represents a global and diverse collection of clinical isolates of MTBC, and includes drug-resistant strains from patients and from in vitro evolution experiments, as well as non-MTBC bacterial genome sequences. Additionally, the genome sequences were chosen to represent a technically diverse collection (Illumina HiSeq 2000, GAIIx and MiSeq). In addition to the genome sequences generated in-house, the test set contains fastq files downloaded from www.sanger.ac.uk, the European Nucleotide Archive (ENA; http://www.ebi.ac.uk/ena/) and the Sequence Read Archive (SRA; http://www.ncbi.nlm.nih.gov/ sra). More information on the fastq files, including accession numbers, can be found in Additional file 2 .

\section{Mapping data for comparison with KvarQ}

To compare KvarQ SNP calls with SNP calls obtained from conventional mapping of fastq short sequencing reads to a reference, we used a previously published computational pipeline [43]. In brief, all short reads were mapped to a hypothetical reconstructed ancestor with BWA, reads were piled up with SAMtools and variants called with BCFtools [51]. Filtering for low quality calls was done with customized scripts. The 206 positions that were used in KvarQ for allele detection were extracted with a Python script and compared to the KvarQ calls in the corresponding sequence. Discrepant results were manually checked with Artemis [52].

\section{KvarQ parameters used}

All KvarQ results were generated using default parameters, i.e. a quality cutoff of 13 , a minimum read length of 25 , a minimum overlap of 25 , a minimum coverage of two reads for allele calls and a maximum of two errors per read. Parameters were adjusted for fastq files with low coverage or low quality values, and are reported in Additional file 2. In case of paired-end sequencing files, only one of two read files was used. Files were manually concatenated in case of low coverage (reported in Additional file 2).

\section{Hardware specification}

KvarQ runs on any system that runs Python and has a POSIX threads implementation (this includes notably Windows, OS X and Linux). Analyses in this study were performed on a Red Hat Enterprise Linux Server release 5.9 (Tikanga) with four six-core AMD Opteron CPUs and 132 GB RAM, using 8 threads.

\section{Ethics statement}

All the work in this study was performed using previously published data (see Additional file 2).

\section{Availability and requirements}

Project name: KvarQ: Targeted and Direct Variant Calling from FastQ Reads of Bacterial Genomes

Project home page: http://www.swisstph.ch/kvarq Operating systems: platform independent; requires Python 2.7, POSIX thread library; pre-compiled packages for OS X (10.6.8 and later) and Windows (7 and later)

Programming Language: Python/C

Other requirements: (none)

Licence: GNU GPL v3

\section{Additional files}

Additional file 1: List of SNPs used as markers for phylogenetic classification of MTBC, and drug resistance markers used in this study.

Additional file 2: List of fastq files used in this study including KvarQ results and additional information.

Competing interests

The authors declare that they have no competing interests.

\section{Authors' contributions}

AS and DS designed and developed the software. AS, DS and SG were responsible for the selection of markers and the compilation of the data set. AS, DS, SB and MC carried out DNA sequencing and data comparison. AS, DS and SG drafted the manuscript. SG coordinated the study. All authors read and approved the final manuscript. 


\section{Acknowledgments}

We thank the anonymous reviewers for their comments. This work was supported by the National Institutes of Health (Al090928), the Swiss National Science Foundation (PP00P3_150750), the European Research Council (309540-EVODRTB), and SystemsX.ch.

Received: 6 February 2014 Accepted: 3 October 2014 Published: 9 October 2014

\section{References}

1. Olsen RJ, Long SW, Musser JM: Bacterial genomics in infectious disease and the clinical pathology laboratory. Arch Pathol Lab Med 2012, 136(11):1414-1422. doi:10.5858/arpa.2012-0025-RA.

2. Bertelli C, Greub G: Rapid bacterial genome sequencing: methods and applications in clinical microbiology. Clin Microbiol Infect 2013, 19(9):803-813. doi:10.1111/1469-0691.12217.

3. Branco M: Bridging genomics technology and biology. Genome Biol 2013, 14(10):312. doi:10.1186/gb4135.

4. Robinson ER, Walker TM, Pallen MJ: Genomics and outbreak investigation: from sequence to consequence. Genome Med 2013, 5(4):36. doi: 10.1186/ gm440.

5. Parkhill J, Wren B: Bacterial epidemiology and biology - lessons from genome sequencing. Genome Biol 2011, 12(10):230. doi:10.1186/gb-201112-10-230.

6. Loman NJ, Constantinidou C, Chan JZM, Halachev M, Sergeant M, Penn CW, Robinson ER, Pallen MJ: High-throughput bacterial genome sequencing: an embarrassment of choice, a world of opportunity. Nat Rev Micro 2012, 10(9):599-606. doi:10.1038/nrmicro2850.

7. Le VTM, Diep BA: Selected insights from application of whole-genome sequencing for outbreak investigations. Curr Opin Crit Care 2013, 19(5):432-439. doi:10.1097/MCC.0b013e3283636b8c.

8. Croucher NJ, Harris SR, Grad YH, Hanage WP: Bacterial genomes in epidemiology-present and future. Philos Trans R Soc Lond B Biol Sci 2013, 368(1614):20120202. doi:10.1098/rstb.2012.0202.

9. Gilmour MW, Graham M, Reimer A, Van Domselaar G: Public health genomics and the new molecular epidemiology of bacterial pathogens. Public Health Genomi 2013, 16(1-2):25-30. doi:10.1159/000342709.

10. Gardy JL: Investigation of disease outbreaks with genome sequencing. Lancet Infect Dis 2013, 13(2):101-102. doi:10.1016/S1473-3099(12)70295-5.

11. Walker TM, Monk P, Smith EG, Peto TEA: Contact investigations for outbreaks of Mycobacterium tuberculosis: advances through whole genome sequencing. Clin Microbiol and Infect 2013, 19(9):796-802. doi:10.1111/1469-0691.12183

12. Didelot $X$, Eyre D, Cule M, Ip C, Ansari M, Griffiths D, Vaughan A, O'Connor L, Golubchik T, Batty E, Piazza P, Wilson D, Bowden R, Donnelly P, Dingle K, Wilcox M, Walker A, APeto T, Harding R: Microevolutionary analysis of Clostridium difficile genomes to investigate transmission. Genome Biol (2012, 13(12):118. doi:10.1186/gb-2012-13-12-r118.

13. Snyder LA, Loman NJ, Faraj LA, Levi K, Weinstock G, Boswell TC, Pallen MJ, Ala'Aldeen DA: Epidemiological investigation of Pseudomonas aeruginosa isolates from a six-year-long hospital outbreak using high-throughput whole genome sequencing. Euro Surveill 2013, 18(42).

14. Snitkin ES, Zelazny AM, Thomas PJ, Stock F, Henderson DK, Palmore TN, Segre JA: Tracking a hospital outbreak of carbapenem-resistant Klebsiella pneumoniae with whole-genome sequencing. Sci Trans/ Med 2012, 4(148):148-116. doi:10.1126/scitransImed.3004129.

15. Lavezzo E, Toppo S, Franchin E, Di Camillo B, Finotello F, Falda M, Manganelli R, Palu G, Barzon L: Genomic comparative analysis and gene function prediction in infectious diseases: application to the investigation of a meningitis outbreak. BMC Infect Dis 2013, 13:554 doi:10.1186/1471-2334-13-554.

16. Köser C, Holden M, Ellington M, Cartwright E, Brown N, Ogilvy-Stuart A, Hsu L, Chewapreecha C, Croucher N, Harris S, Sanders M, Enright MC, Dougan G, Bentley SD, Parkhill J, Fraser LJ, Betley JR, Schulz-Trieglaff OB, Smith GP, Peacock SJ: Rapid whole-genome sequencing for investigation of a neonatal MRSA outbreak. New Engl J Med 2012, 366(24):2267-2275. doi:10.1056/NEJMoa1109910

17. Castillo-Ramirez S, Corander J, Marttinen P, Aldeljawi M, Hanage W, Westh H, Boye K, Gulay Z, Bentley S, Parkhill J, Holden M, Feil E: Phylogeographic variation in recombination rates within a global clone of methicillin- resistant Staphylococcus aureus. Genome Biol 2012, 13(12):126 doi:10.1186/gb-2012-13-12-r126.

18. Gardy JL, Johnston JC, Ho Sui SJ, Cook VJ, Shah L, Brodkin E, Rempel S, Moore R, Zhao Y, Holt R, Varhol R, Birol I, Lem M, Sharma MK, Elwood K, Jones SJM, Brinkman FSL, Brunham RC, Tang P: Whole-genome sequencing and social-network analysis of a tuberculosis outbreak. New Engl J Med 2011, 364(8):730-739. doi: 10.1056/NEJMoa1003176.

19. Bryant JM, Schürch $A C$, van Deutekom $H$, Harris SR, de Beer JL, de Jager $V_{\text {, }}$ Kremer K, van Hijum SA, Siezen RJ, Borgdorff M, Bentley SD, Parkhill J, van Soolingen D: Inferring patient to patient transmission of Mycobacterium tuberculosis from whole genome sequencing data. BMC Infect Dis 2013, 13(1):110. doi:10.1186/1471-2334-13-110.

20. Roetzer A, Diel R, Kohl TA, Rückert C, Nübel U, Blom J, Wirth T, Jaenicke $S$, Schuback S, Rüsch-Gerdes S, Supply P, Kalinowski J, Niemann S: Whole genome sequencing versus traditional genotyping for investigation of a Mycobacterium tuberculosis outbreak: a longitudinal molecular epidemiological study. PLoS Med 2013, 10(2):1001387. doi:10.1371/journal. pmed.1001387.

21. Walker TM, Ip CLC, Harrell RH, Evans JT, Kapatai G, Dedicoat MJ, Eyre DW, Wilson DJ, Hawkey PM, Crook DW, Parkhill J, Harris D, Walker AS, Bowden R, Monk P, Smith EG, Peto TEA: Whole-genome sequencing to delineate Mycobacterium tuberculosis outbreaks: a retrospective observational study. Lancet Infect Dis 2013, 13(2):137-146. doi:10.1016/S1473-3099(12) 70277-3

22. Mather AE, Reid SWJ, Maskell DJ, Parkhill J, Fookes MC, Harris SR, Brown DJ, Coia JE, Mulvey MR, Gilmour MW, Petrovska L, de Pinna E, Kuroda M, Akiba M, Izumiya H, Connor TR, Suchard MA, Lemey P, Mellor DJ, Haydon DT, Thomson NR: Distinguishable epidemics of multidrug-resistant Salmonella typhimurium dt104 in different hosts. Science 2013, 341(6153):1514-1517. doi:10.1126/ science.1240578.

23. Drobniewski F, Nikolayevskyy V, Maxeiner H, Balabanova Y, Casali N, Kontsevaya I, Ignatyeva O: Rapid diagnostics of tuberculosis and drug resistance in the industrialized world: clinical and public health benefits and barriers to implementation. BMC Med 2013, 11:190. doi:10.1186/17417015-11-190.

24. Chen Y, Mukherjee S, Hoffmann M, Kotewicz ML, Young S, Abbott J, Luo Y, Davidson MK, Allard M, McDermott P, Zhao S: Whole-genome sequencing of gentamicin-resistant Campylobacter coli isolated from u.s. retail meats reveals novel plasmid-mediated aminoglycoside resistance genes. Antimicrob Agents Chemother 2013, 57(11):5398-5405. doi:10.1128/AAC.00669-13.

25. Köser CU, Bryant JM, Becq J, Török ME, Ellington MJ, Marti-Renom MA, Carmichael AJ, Parkhill J, Smith GP, Peacock SJ: Whole-genome sequencing for rapid susceptibility testing of M. tuberculosis. New Engl J Med 2013, 369(3):290-292. doi:10.1056/NEJMc1215305.

26. Paterson GK, Morgan FJE, Harrison EM, Cartwright EJP, Torok ME, Zadoks RN, Parkhill J, Peacock SJ, Holmes MA: Prevalence and characterization of human mecc methicillin-resistant Staphylococcus aureus isolates in England. J Antimicrob Chemother 2013. doi:10.1093/jac/dkt462.

27. Zankari E, Hasman H, Kaas RS, Seyfarth AM, Agersø Y, Lund O, Larsen MV, Aarestrup FM: Genotyping using whole-genome sequencing is a realistic alternative to surveillance based on phenotypic antimicrobial susceptibility testing. Antimicrob Chemother 2013, 68(4):771-777. doi:10.1093/jac/dks496.

28. Török ME, Peacock SJ: Rapid whole-genome sequencing of bacterial pathogens in the clinical microbiology laboratory-pipe dream or reality? Antimicrob Chemother 2012, 67(10):2307-2308.

29. Pallen MJ, Loman NJ, Penn CW: High-throughput sequencing and clinical microbiology: progress, opportunities and challenges. Curr Opin Microbiol 2010, 13(5):625-631. doi:10.1016/j.mib.2010.08.003.

30. Stucki D, Gagneux S: Single nucleotide polymorphisms in Mycobacterium tuberculosis and the need for a curated database. Tuberculosis 2012 doi:10.1016/j.tube.2012.11.002

31. Kruczkiewicz P, Mutschall S, Barker D, Thomas J, Van Domselaar G, Gannon VP, Carrillo CD, Taboada EN: MIST: a Tool for Rapid in Silico Generation of Molecular Data from Bacterial Genome Sequences. https://bytebucket. org/peterk87/microbialinsilicotyper/wiki/mist_paper.pdf.

32. Jolley K, Maiden M, BIGSdb: Scalable analysis of bacterial genome variation at the population level. BMC Bioinformatics 2010, 11(1):595. doi:10.1186/1471-2105-11-595.

33. Inouye $M$, Conway $T$, Zobel J, Holt $\mathrm{K}$ : Short read sequence typing (SRST): multi-locus sequence types from short reads. BMC Genomics 2012, 13(1):338. doi:10.1186/1471-2164-13-338 
34. Ramaswamy S, Musser JM: Molecular genetic basis of antimicrobial agent resistance in Mycobacterium tuberculosis: 1998 update. Tubercle Lung Dis 1998, 79(1):3-29. doi:10.1054/tuld.1998.0002.

35. Riska PF, Jacobs WR, Alland D: Molecular determinants of drug resistance in tuberculosis. Tubercle Lung Dis 2000, 4(2 Suppl 1):4-10.

36. Rodwell TC, Valafar F, Douglas J, Qian L, Garfein RS, Chawla A, Torres J, Zadorozhny V, Soo Kim M, Hoshide M, Catanzaro D, Jackson L, Lin G, Desmond E, Rodrigues C, Eisenach K, Victor TC, Ismail N, Crudu V, Gle MT, Catanzaro A: Predicting extensively drug-resistant tuberculosis (XDR-TB) phenotypes with genetic mutations. J Clin Microbio/ 2013. doi:10.1128/ JCM.02701-13.

37. Hershberg R, Lipatov M, Small PM, Sheffer H, Niemann S, Homolka S, Roach JC, Kremer K, Petrov DA, Feldman MW, Gagneux S: High functional diversity in Mycobacterium tuberculosis driven by genetic drift and human demography. PLOS Biol 2008, 6(12):311. doi:10.1371/journal. pbio.0060311.

38. Comas I, Chakravartti J, Small PM, Galagan J, Niemann S, Kremer K, Ernst JD, Gagneux S: Human T cell epitopes of Mycobacterium tuberculosis are evolutionarily hyperconserved. Nat Genet 2010, 42(6):498-503. doi:10.1038/ng.590.

39. Coscolla M, Gagneux S: Does M. tuberculosis genomic diversity explain disease diversity? Drug disc today 2010, 7(1):43-59. doi:10.1016/j. ddmec.2010.09.004.

40. Stucki D, Malla B, Hostettler S, Huna T, Feldmann J, Yeboah-Manu D, Borrell S, Fenner L, Comas I, Coscollà M, Gagneux S: Two new rapid SNP-Typing methods for classifying Mycobacterium tuberculosis complex into the main phylogenetic lineages. PLoS One 2012, 7(7):41253. doi:10.1371/journal. pone.0041253.

41. Comas I, Coscolla M, Luo T, Borrell S, Holt KE, Kato-Maeda M, Parkhill J, Malla B, Berg S, Thwaites G, Yeboah-Manu D, Bothamley G, Mei J, Wei L, Bentley S, Harris SR, Niemann S, Diel R, Aseffa A, Gao Q, Young D, Gagneux S: Out-of-africa migration and neolithic coexpansion of Mycobacterium tuberculosis with modern humans. Nat Genet 2013, 45(10):1176-1182. doi:10.1038/ng.2744.

42. Comas I, Homolka S, Niemann S, Gagneux S: Genotyping of genetically monomorphic bacteria: DNA sequencing in Mycobacterium tuberculosis highlights the limitations of current methodologies. PLoS One 2009, 4(11):7815. doi:10.1371/journal.pone.0007815.

43. Coscolla M, Lewin A, Metzger S, Maetz-Rennsing K, Calvignac-Spencer S, Nitsche A, Dabrowski PW, Radonic A, Niemann S, Parkhill J, Couacy-Hymann E, Feldman J, Comas I, Boesch C, Gagneux S, Leendertz FH: Novel Mycobacterium tuberculosis complex isolate from a wild chimpanzee. Emerg Infect Dis 2013, 19(6):969-976. doi:10.3201/eid1906.121012.

44. Cole ST, Brosch R, Parkhill J, Garnier T, Churcher C, Harris D, Gordon SV, Eiglmeier K, Gas S, Barry CE, Tekaia F, Badcock K, Basham D, Brown D, Chillingworth T, Connor R, Davies R, Devlin K, Feltwell T, Gentles S, Hamlin N, Holroyd S, Hornsby T, Jagels K, Krogh A, McLean J, Moule S, Murphy L, Oliver K, Osborne J, et al: Deciphering the biology of Mycobacterium tuberculosis from the complete genome sequence. Nature 1998, 393(6685):537-544. doi:10.1038/31159.

45. Warner DF, Mizrahi V: Complex genetics of drug resistance in Mycobacterium tuberculosis. Nat Genet 2013, 45(10):1107-1108. doi:10.1038/ng.2769.

46. Sandgren A, Strong M, Muthukrishnan P, Weiner BK, Church GM, Murray MB: Tuberculosis drug resistance mutation database. PLoS Med 2009, 6(2):1000002. doi:10.1371/journal.pmed.1000002.

47. Comas I, Borrell S, Roetzer A, Rose G, Malla B, Kato-Maeda M, Galagan J, Niemann S, Gagneux S: Whole-genome sequencing of rifampicin-resistant Mycobacterium tuberculosis strains identifies compensatory mutations in RNA polymerase genes. Nat Genet 2012, 44(1):106-110. doi:10.1038/ ng.1038.

48. Takiff HE, Salazar L, Guerrero C, Philipp W, Huang WM, Kreiswirth B, Cole ST, Jacobs WR, Telenti A: Cloning and nucleotide sequence of Mycobacterium tuberculosis gyrA and gyrB genes and detection of quinolone resistance mutations. Antimicrob Chemother 1994, 38(4):773-780. doi:10.1128/AAC.38.4.773

49. Camus J-C, Pryor MJ, Médigue C, Cole ST: Re-annotation of the genome sequence of Mycobacterium tuberculosis H37Rv. Microbiology 2002, 148(Pt 10):2967-2973.

50. Lew JM, Kapopoulou A, Jones LM, Cole ST: TubercuList - 10 years after. Tuberculosis 2011, 91(1):1-7. doi:10.1016/j.tube.2010.09.008.
51. Li H, Durbin R: Fast and accurate short read alignment with burrowswheeler transform. Bioinformatics 2009, 25(14):1754-1760. doi:10.1093/ bioinformatics/btp324.

52. Rutherford K, Parkhill J, Crook J, Horsnell T, Rice P, Rajandream MA, Barrell B: Artemis: sequence visualization and annotation. Bioinformatics 2000, 16(10):944-945.

doi:10.1186/1471-2164-15-881

Cite this article as: Steiner et al:: KvarQ: targeted and direct variant calling from fasta reads of bacterial genomes. BMC Genomics 2014 15:881.

\section{Submit your next manuscript to BioMed Central and take full advantage of:}

- Convenient online submission

- Thorough peer review

- No space constraints or color figure charges

- Immediate publication on acceptance

- Inclusion in PubMed, CAS, Scopus and Google Scholar

- Research which is freely available for redistribution

Submit your manuscript at www.biomedcentral.com/submit
C) Biomed Central 